\title{
A new role for plant R2R3-MYB transcription factors in cell cycle regulation
}

\author{
Eleonora Cominelli ${ }^{1}$, Chiara Tonelli ${ }^{1}$ \\ ${ }^{1}$ Dipartimento di Scienze Biomolecolari e Biotecnologie, Università degli Studi di Milano, Via Celoria 26, 20133 Milano, Italy \\ Cell Research (2009) 19:1231-1232. doi: 10.1038/cr.2009.123; published online 2 November 2009
}

MYB proteins are transcription factors present in all eukaryotes, sharing a common DNA-binding domain that consists of one to three imperfect helix-helix-turn-helix repeats of about 50 amino acids, called R1, R2, and R3 respectively [1]. In animals and yeast these proteins represent a small gene family [1]. Animal R1R2R3-MYB proteins have been described for their role in cell cycle regulation mainly at the $\mathrm{G} 1 / \mathrm{S}$, but also at the $\mathrm{G} 2 / \mathrm{M}$ transition, as firstly demonstrated in Drosophila [2].

In plants the MYB superfamily constitutes the most abundant group of transcription factors. From an analysis of the complete Arabidopsis genome sequence 198 genes in the MYB superfamily were identified, among them 126 are $R 2 R 3-M Y B, 5$ are $R 1 R 2 R 3-M Y B$, 64 are $M Y B$-related and 3 are atypical $M Y B$ genes [1]. Plant R1R2R3-MYB proteins, whose repeats are more closely related to the repeats present in vertebrate MYB proteins than to the repeats from plant R2R3-MYB proteins, play a role in the regulation of genes involved in the cell cycle G2/M phase, as shown in tobacco and Arabidopsis [3, 4]. They bind to mitosis-specific activator (MSA) elements, present in most promoter sequences of genes specifically expressed in the G2/M phase in Arabidopsis [5].

Correspondence: Eleonora Cominelli Tel: +390250315009; Fax: +390250315044

E-mail: eleonora.cominelli@unimi.it
Also the MYB-related CDC5-like proteins, characterized in different species (yeast, animal and AtCDC5 in Arabidopsis), harboring a MYB domain with two repeats only distantly related to those of the R2R3-type MYB proteins, control the G2/M transition of the cell cycle [6].

Different members of the most highly represented R2R3-MYB subfamily have been characterized for their roles in the regulation of plant-specific processes, such as phenylpropanoid metabolism, determination of specialized cell morphology, and responses to biotic and abiotic stresses [1]. Until the beginning of this year no clear involvement of R2R3-MYB subfamily members in the regulation of cell cycle was reported, although their role in the local control of cell proliferation was suggested by some authors [7 and references therein]. This year two papers reported a role of two members of this Arabidopsis subfamily in the regulation of the cell cycle. These are AtMYB59, which is involved in root growth [8] and DUO1 (AtMYB125), encoding a male germline-specific protein [9].

$\mathrm{Mu}$ et al. [8] reported a detailed analysis of AtMYB59 in Cell Research. AtMYB59 is preferentially expressed in roots and encodes a protein that has transcriptional activation activity. They found that the expression of AtMYB59 transcription factor in transformed yeast cells altered DNA synthesis and/ or chromosome separation, affecting, as a consequence, the cell division process. In fact the proliferation of AtMYB59-transformed cells was inhibited and the resulting yeast cells were about threefold longer than the control cells and more than $20 \%$ of the AtMYB59-transformed cells had double or abnormal nuclei. The authors found that also the DNA content in the yeast cells was altered during the cell division and there was a high percentage of aneuploid and apoptotic cells. Cell division in yeast was affected probably due to the inability to form a cell plate between the two daughter cells or due to the interference of the AtMYB59 protein with the activity of the endogenous CDC5, a MYB-related transcription factor involved in cell cycle progression, as previously mentioned.

The authors demonstrated that the AtMYB59 gene is preferentially expressed during the $\mathrm{S}$ and $\mathrm{S}$ to $\mathrm{G} 2$ phases in an Arabidopsis suspension culture. As the AtMYB59 gene is preferentially expressed in roots, Mu et al. investigated the phenotype of this organ in the myb59-1 knock-out mutant and in AtMYB59-overexpressing lines. They showed an opposite phenotype in the primary root length. In the mutant the root was longer than in the wild-type, while in the overexpressing lines the root was shorter. These phenotypes do not depend on differences in root cell structure or size, but on differences in the percentage of cells at each cell cycle phase with the mutant having only $14 \%$ 
of cells at metaphase, the overexpressing line $54 \%$ and the wild-type $27 \%$.

One of the probable targets of the AtMYB59 transcription factor is the $C Y C B 1 ; 1$ gene coding for a cyclin $\mathrm{B}$, a regulatory subunit of a cyclin dependent kinase that has a critical role in $\mathrm{G} 2 / \mathrm{M}$ progression of the cell cycle [10]. In the AtMYB59-overexpressing line $C Y C B 1 ; 1$ gene expression was highly up-regulated, but its expression was only slightly reduced in the myb59-1 mutant, indicating only a partial role of AtMYB59 in the regulation of this cyclin. The AtMYB59 protein binds to some potential MYB-binding elements in the $C Y C B 1 ; 1$ gene promoter, but not to the MSA element. Conversely the corresponding tobacco MSA element in the $C Y C B 1 ; 1$ promoter is bound by the R1R2R3-MYB NtmybA1 and NtmybA2 factors that act as positive regulators and by NtmybB working as a repressor [3]. AtMYB59 might control the expression of $C Y C B 1 ; 1$ and maybe other G2/M phase-specific genes, through its binding to the elements identified by $\mathrm{Mu}$ et al. in a cooperative way with one of the R1R2R3-MYB proteins able to bind to the MSA element [4]. Another possibility is that AtMYB59 acts in a partially redundant fasion with another R2R3-MYB not yet characterized, or with the putative At2g03470 MYB transcription factor, identified as a member of a complex binding to promoter elements of the $C Y C B 1 ; 1$ gene, in combination with transcription factors belonging to other families, such as the HYP protein, which contains a leucine zipper and MYC-type dimerization domains [10].

In another recent paper Brownfield et al. showed that the Arabidopsis male germline-specific DUO1 gene, is required for correct male germline differentiation and for male germline cell division, controlling the expression of the $\mathrm{G} 2 / \mathrm{M}$ regulator $C Y C B 1 ; 1$ [9]. Expression of the cyclin $\mathrm{B}$ as a chimeric gene under the control of the DUO1 promoter can partially rescue defective germ cell division in the duol mutant.

Therefore the recent papers on AtMYB59 function in the root [8] and DUO1 role in the male germline [9] and the hypothesis of involvement of other R2R3MYB members in the local control of cell proliferation [7 and references therein] highlight that R2R3-MYB transcription factors also have a role in the regulation of the cell cycle. Probably in the near future a role in this process will be elucidated for other members of this large subfamily. With the knowledge obtained so far we can speculate that plants have evolved diverse R2R3-MYB proteins to regulate cell cycle progression in an organ-, tissue- or cell-specific way.

\section{Acknowledgments}

We thank Martin Kater (Università degli Studi di Milano) for comments on the manuscript.

\section{References}

1 Chen Y, Yang X, He K, et al. The MYB transcription factor superfamily of Arabidopsis: expression analysis and phylogenetic comparison with the rice MYB family. Plant Mol Biol 2006; 60:107-124.

2 Okada M, Akimaru H, Hou DX, Takahashi T, Ishii S. Myb controls G(2)/M progression by inducing cyclin $\mathrm{B}$ expression in the Drosophila eye imaginal disc. EMBO J 2002; 21:675-684.
3 Ito $\mathrm{M}$, Araki S, Matsunaga S, et al. G2/M-phase-specific transcription during the plant cell cycle is mediated by c-Myb-like transcription factors. Plant Cell 2001; 13:1891-1905.

4 Haga N, Kato K, Murase M, et al. R1R2R3-Myb proteins positively regulate cytokinesis through activation of KNOLLE transcription in Arabidopsis thaliana. Development 2007; 134:11011110.

5 Menges M, de Jager SM, Gruissem W, Murray JA. Global analysis of the core cell cycle regulators of Arabidopsis identifies novel genes, reveals multiple and highly specific profiles of expression and provides a coherent model for plant cell cycle control. Plant J2005; 41:546566.

6 Lin Z, Yin K, Zhu D, Chen Z, Gu H, Qu LJ. AtCDC5 regulates the G2 to M transition of the cell cycle and is critical for the function of Arabidopsis shoot apical meristem. Cell Res 2007; 17:815-828.

7 Petroni K, Falasca G, Calvenzani V, et al. The AtMYB11 gene from Arabidopsis is expressed in meristematic cells and modulates growth in planta and organogenesis in vitro. J Exp Bot 2008; 59:1201-1213.

$8 \mathrm{Mu}$ RL, Cao YR, Liu YF, et al. An R2R3-type transcription factor gene AtMYB59 regulates root growth and cell cycle progression in Arabidopsis. Cell Res 2009; 19:1291-1304.

9 Brownfield L, Hafidh S, Borg M, Sidorova A, Mori T, Twell D. A plant germline-specific integrator of sperm specification and cell cycle progression. PLoS Genet 2009; 5: e1000430. DOI: 10.1471/journal.pgen. 1000430

10 Planchais S, Perennes C, Glab N, Mironov V, Inzé D, Bergounioux C. Characterization of $c i s$-acting element involved in cell cycle phase-independent activation of Arath; $C y c B 1 ; 1$ transcription and identification of putative regulatory proteins. Plant Mol Biol 2002; 50:111127. 